\title{
ABOMASAL DISPLACEMENT IN COWS - MEASUREMENT OF OXYGEN SATURATION OF THE ABOMASAL WALL
}

\author{
Th.Wittek and M. Fürll \\ Medizinische Tierklinik, University of Leipzig \\ An den Tierkliniken 11, 04103 Leipzig, Germany, \\ E-mail: wittek@vetmed.uni-leipzig.de
}

The monitoring of intestinal oxygen saturation by means of a pulse oximeter is routinely applied in human medicine during surgery of the gastrointestinal tract to evaluate the disturbance of perfusion and assess tissue viability. To some extent, this technique has been applied in veterinary medicine. The purpose of this study was to test the technique in cattle and to describe the influence of gas volume and intraluminal pressure of the displaced abomasum on oxygen saturation of the organ.

The study was performed on 35 cows suffering from left or right abomasal displacement (LDA, 20 cows; RDA (torsions $\geq 180^{\circ}, 15$ cows). The cows, ages 2,5 to 7 years, were a cross between the local dairy cow breed (Schwarzbuntes Milchrind) and Holstein-Friesian.

A pulse oximeter (CardiocapII-CG) was used to monitor oxygen saturation on the displaced abomasum and, as a comparison, on the rumen. The pulse oximetry probe was attached to the highest point of the gas-filled vault of the abomasum and the most caudal aspect of the dorsocaudal ruminal sack (transmission pulse oximetry). The gas pressure was measured by cannulating a needle (diameter 2,1 $\mathrm{mm}$,) and a non-compressible tube connected to the electronic manometer (BMT 401). The gas volume was measured by aspirating the complete gas volume with an aspirator (GF 80) and pipelining the gas into a water-filled container to displace the water. The displaced water volume was registered as the indirect gas volume.

The oxygen saturation of the abomasal wall was lower in cows with right abomasal volvulus $(79,4$ $\%)$ than in cows with LDA $(83,8 \%)$. In comparison to the abomasum, the oxygen saturation of the dorsal rumen was $89,1 \%$ in cows with LDA and $89,5 \%$ in cows with RDA. The oxygen saturation in cows with LDA did not correlate to the gas pressure nor to the gas volume of the displaced abomasum. In cows with RDA, the oxygen saturation correlated negatively to the gas volume $\left(r_{s}=-0,16\right)$ and to the gas pressure $\left(r_{s}=-0,15\right)$.

The average intraluminal pressure and the abomasal gas volume in cows with LDA $(6,04 \pm 2,16$ mmHg respectively 7,98 $\pm 2,041)$ was significantly lower than in cows with $\operatorname{RDA}(12,86 \pm 5,60$ $\mathrm{mmHg}$ respectively $11,53 \pm 3,861$ ). The weak correlation between gas pressure and gas volume can be a result of other factors which influence the intraabdominal pressure and volume, like ruminal content or tension of the abdominal wall. Factors causing the relatively weak correlation between gas pressure and volume on one hand and the oxygen saturation on the other hand might be the unknown time lapse (in most cases) between the beginning of the abomasal displacement and the admission into the clinic, the imprecise degree of disturbance in the motility and the unknown amount of fluid in the abomasum.

The application of pulse oximetry to the gastrointestinal tract in cattle during laparotomy is feasible and requires a short amount of time. In the cows in this study, the measured oxygen saturation indicated that the perfusion of the displaced abomasum was altered in LDA as well as in RDA. Probable 
reasons for this disturbance are the impact of pressure caused by the content of gas and fluid to the abomasal wall, the compression of the blood vessels due to the distension of the abomasal wall and the influence of the displacement or torsion of the abomasum itself on the the vessels in the omentum. Severe disturbances in perfusion have to be expected in cases of right abomasal displacement where severe torsions of the abomasum are present. This technique can be useful to establish a prognosis and metaphylactic therapeutic efforts to prevent reperfusions injuries, paralytic postoperative ileus, and abomasal atony.

\title{
ANTIBIOTIC SUSCEPTIBILITY AND RESISTANCE OF STAPHYLOCOCCUS CHROMOGENES FROM BOVINE MASTITIS
}

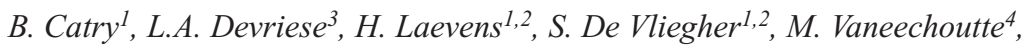 \\ G. Opsomer ${ }^{1}$, and A. de Kruif ${ }^{1}$ \\ ${ }^{1}$ Department of Reproduction, Obstetrics, and Herd Health, ${ }^{2}$ Veterinary Epidemiology Unit, ${ }^{3}$ Department of \\ Bacteriology, Faculty of Veterinary Medicine, Ghent University, Merelbeke, Belgium; ${ }^{4}$ Department of \\ Chemistry, Microbiology and Immunology, Ghent University Hospital, Ghent, Belgium.
}

An unselected series of seventy-six strains isolated from subclinical mastitis from forty-four dairy herds in Flanders, Belgium, tentatively identified as Staphylococcus chromogenes was examined for confirmation of identification and antibiotic susceptibility patterns.

The presumptive identification was only based upon complete absence of hemolysis and a typically weakly positive DNase test, usually with yellow pigmentation. A confirmatory identification was performed by tRNA intergenic spacer PCR as described by Maes et al. (1997, J. Clin. Microbiol. 35, 2477-2481). Antibiotic resistance profiles to several antibiotics were determined as MIC's (Minimal Inhibitory Concentrations) using agar plate dilution on Mueller-Hinton II medium (BBL) according to NCCLS guidelines. The presence of $\beta$-lactamase was tested by visual inspection of the margins of penicillin disk diffusion zones (positive when heaped up with fully grown colonies), and the nitrocefin test (Oxoid). mecA Gene determination with PCR was carried out only on penicillin-resistant strains in order to detect methicillin-type resistance (resistance to ß-lactamase-resistant penicillins and cefalosporins).

Confirmation of identification by PCR resulted in sixty-two strains of $S$. chromogenes, three strains of Staphylococcus sciuri, one remarkable Staphylococcus aureus (described below), and ten unidentified strains. The antibiotic resistance profiles of the $S$. chromogenes are described as $\mathrm{MIC}_{90}$ and $\mathrm{MIC}_{50}$ and given in Table 1. 
Table 1. Susceptibility of S. chromogenes from bovine mastitis $(n=62)$.

\begin{tabular}{lccccccccc}
\hline & Peni G & Cloxa & Tetra & Tylo & Ery & Linco & Genta & Neo & Enro \\
\hline $\mathrm{MIC}_{90}$ & 0.12 & 0.50 & 0.25 & 1.00 & 0.25 & 1.00 & $\leq 0.06$ & 0.25 & 0.12 \\
$\mathrm{MIC}_{50}$ & $\leq 0.06$ & 0.25 & 0.25 & 0.50 & 0.25 & 0.50 & $\leq 0.06$ & 0.25 & 0.12 \\
\hline
\end{tabular}

Twenty-four strains of $S$. chromogenes were $\beta$-lactamase-positive and the presence of $\beta$-lactamase was associated with pigmentation (Fisher Exact test: $\mathrm{P}=0.002$ ). Of the fifty-one yellow-pigmented strains only fifteen were b-lactamase positive, while nine out of eleven white colonies were $\beta$-lactamase positive. The results of visual inspection of the margins of penicillin inhibition zones completely corresponded to those of nitrocefin tests. The mecA gene, indicating methicilline resistance, was found in two strains. One of these strains was not pigmented, DNase negative, non-hemolytic, tube coagulase-negative, clumping factor-negative, lipase-negative, lecithinase-negative and novobiocin-resistant and proved to be a highly atypical $S$. aureus. This was confirmed by tRNA intergenic spacer PCR, femB gene determination, and acriflavine $(8 \mu \mathrm{g} / \mathrm{ml})$ resistance. A second strain was identified as $S$. sciuri.

The dilution method, with the NCCLS recommended low number of cells $\left(10^{5}\right)$, is not suitable to determine susceptibility and resistance to penicillins and cephalosporins in staphylococci. In comparison with reports of the susceptibility patterns of $S$. aureus, $S$. chromogenes appeared to have a similar antibiotic resistance profile, though the resistance rates may be somewhat lower.

\title{
PREVALENCE OF TEAT APEX COLONIZATIONS BY S. CHROMOGENES IN YOUNG AND PRIMIGRAVID DAIRY HEIFERS
}

\author{
${ }^{1,2}$ S.De Vliegher, ${ }^{1,2}$ H.Laevens, ${ }^{1}$ G.Opsomer, ${ }^{1}$ B.Catry, ${ }^{1}$ J.Leroy, ${ }^{1}$ A.de Kruif \\ ${ }^{1}$ Department of Reproduction, Obstetrics and Herd Health, ${ }^{2}$ Veterinary Epidemiology Unit, \\ Faculty of Veterinary Medicine, Ghent University, 9820 Merelbeke, Belgium
}

Little attention has been paid to intramammary infections (IMI) in dairy heifers as their non-lactating udders have traditionally been considered as uninfected. However, high prevalences of IMI in non-lactating and freshly calved heifers, mainly caused by Non-aureus staphylococci (NAS), were reported (1). Prevalence of teat apex and teat skin colonizations is also high. They are mainly caused by the same species (3). The aim of this study was to examine the prevalence of teat apex colonization with Staphylococcus chromogenes, one of the most prevalent NAS, in young and primigravid dairy heifers, and the effect of age on this prevalence.

In a cross-sectional study, teat ends $(n=492)$ were sampled from in total 123 heifers, on eight dairy herds. The age of the heifers varied from 8 to 34 months. The heifers were immobilized and the teat apexes were cleansed with a sterile cloth, sampled with a swab (Cultiplast $\left.{ }^{\circledR}\right)$ and put on to a transport medium. All swabs were streaked on a blood agar plate and incubated aerobically at $37^{\circ} \mathrm{C}$. Bac- 
terial growth was recorded after 24 and 48 hours of incubation. Identification was performed as described by Devriese et al. (2). A teat apex was classified as infected with S. chromogenes when at least 8 colony forming units were isolated. Prevalences were calculated as the percentage of heifers with at least one infected teat apex and as the percentage of infected teat apexes. The mean number of infected teat apexes per heifer was also calculated. Logistic regression was used to analyze the effect of age of the heifers on prevalence of $S$. chromogenes. Odds Ratio's (OR) per age group, using the youngest age group as reference, were calculated (95\% Confidence Intervals-CI)(SPSS 10.0).

The mean age of the heifers at the moment of sampling was 18.7 months. Overall, $20.3 \%$ of the heifers had S. chromogenes infected teat apexes, while $9.7 \%$ of all teat apexes were infected with $S$. chromogenes. There was a significant influence of age class on the prevalence of infected heifers $(\mathrm{P}<0.05)$. Prevalences of infected heifers (with $\mathrm{OR}$ ), prevalences of infected teat apexes per age class, and mean number of infected teat apexes per heifer are presented in Table 1.

Table 1: Prevalences of $S$. chromogenes infected heifers and S. chromogenes infected teat apexes per age class and mean number of infected teat apexes per heifer.

\begin{tabular}{rccccc}
\hline Age class & $\begin{array}{c}\mathrm{N} \\
\text { (heifers) }\end{array}$ & $\begin{array}{c}\text { Prevalence } \\
(\% \text { of heifers) }\end{array}$ & OR (95\% CI) & $\begin{array}{c}\text { Prevalence } \\
(\% \text { of apexes })\end{array}$ & $\begin{array}{c}\text { Mean infected } \\
\text { number of } \\
\text { infected teat } \\
\text { apexes per heifer }\end{array}$ \\
\hline $8-12$ months & 22 & $9,1 \%$ & & $4,5 \%$ & 2 \\
$>12-20$ months & 52 & $7,7 \%$ & $0,8(0,1-4,9)$ & $3,4 \%$ & 1,75 \\
$>20-28$ months & 37 & $35,1 \%$ & $5,4(1,1-26,9)$ & $16,2 \%$ & 1,85 \\
$>28-34$ months & 12 & $50,0 \%$ & $10,0(1,6-63,1)$ & $27,1 \%$ & 2,16 \\
\hline
\end{tabular}

This study shows that heifers older than 20 months of age run a higher risk of having $S$. chromogenes infected teat apexes than younger ones. Further, longitudinal studies will reveal whether IMI post partum can be predicted by $S$. chromogenes teat apex colonizations and whether there is a quantitative effect. If an association exists, possible risk factors for teat apex colonization can be identified and can be used to reduce the high prevalence of IMI in freshly calved dairy heifers.

1. Boddie RL, Nickerson SC, Owens WE, Watts JL. (1987). Udder microflora in nonlactating heifers. AgriPractice 8, 22-25.

2. Devriese LA, Laevens H, Haesebrouck F, Hommez J (1994). A simple identification scheme for coagulase negative Staphylococci from bovine mastitis. Res. Vet.Sci. 57, 240-244.

3. White DG, Harmon RJ, Matos JE, Langlois BE. (1989). Isolation and identification of coagulase-negative Staphylococcus species from bovine body sites and streak canals of nulliparous heifers. J Dairy Sci. 72, 18861892. 